\title{
Régimes institutionnels de ressources et théorie de la régulation
}

\author{
Frédéric Varone \\ Stéphane Nahrath \\ Jean-David Gerber ${ }^{1}$
}

\section{Résumé}

Cet article postule que la combinaison d'une approche en termes de science politique (analyse des politiques publiques) et d'économie institutionnelle des ressources (théorie des droits de propriété) permet l'identification des dimensions régulatrices les plus importantes permettant d'expliquer l'usage (non) durable des ressources naturelles et culturelles. Sur cette base, il développe un cadre d'analyse novateur, celui des régimes institutionnels de ressources (RIR), qui est ensuite confronté au corpus de la théorie française de la régulation (TR, telle que formalisée notamment par M. Aglietta, R. Boyer et A. Lipietz) de manière à identifier, aussi bien les convergences, que les complémentarités entre les RIR et la TR. Nous développons ainsi l'idée que l'une des contributions majeures des RIR à la TR sous l'angle de la durabilité consiste dans sa capacité à décrire les différentes configurations de régimes de ressource et à expliquer, voire prévoir, leurs effets sur la durabilité de la ressource.

\begin{abstract}
This article postulates that an approach combining political science (policy analysis) and institutional economics (property rights theory) enables the identification of the most relevant regulatory dimensions that explain the (un)sustainable uses of natural and cultural resources. From this starting point, it develops an innovative theoretical framework, that of the institutional resource regime (IRR). This framework is then compared with the corpus of the French theory of regulation (TR, as formalized by M. Aglietta, R. Boyer and A. Lipietz). We develop the idea that one of the major contributions of the IRR framework to the TR from a sustainability perspective is its ability to describe the different configurations of resource regimes and thus to explain, and even predict, their effects on the sustainability of a resource.
\end{abstract}

Mots clés

Régime institutionnel de ressource, analyse des politiques publiques, économie institutionnelle, droit de propriété, gestion durable des ressources

Key words

Institutional resource, regime policy analysis, institutional economics, property rights, resource use, sustainable resource management

Codes JEL: A12, K11, K32, N50, N54, O13, P14, Q15, Q2, Q5, R52

\section{Pour citer cet article}

Frédéric Varone, Stéphane Nahrath, Jean-David Gerber, "Régimes institutionnels de ressources et théorie de la régulation ", Revue de la régulation, $\mathrm{n}^{\circ} 2$, Varia, [En ligne], mis en ligne le 31 janvier 2008. URL : http://regulation.revues.org/ consulté le 31 janvier 2008.

1 frederic.varone@politic.unige.ch ； stephane.nahrath@idheap.unil.ch ; jeandavid.gerber@idheap.unil.ch 


\title{
Régimes institutionnels de ressources et théorie de la régulation
}

\author{
Frédéric Varone \\ Stéphane Nahrath \\ Jean-David Gerber
}

\section{Introduction}

Le concept de développement durable (DD) s'est frayé un cheminement institutionnel depuis une quinzaine d'années (WCED 1987) et figure aujourd'hui parmi les priorités de l'agenda politique de nombreux gouvernements. Néanmoins, force est de constater que, malgré la mise en œuvre volontariste de multiples politiques de protection, la dégradation de l'environnement et la surexploitation de la plupart des ressources naturelles demeurent des problèmes lancinants (McNeill 2001). Ces politiques sectorielles (par exemple de lutte contre la pollution atmosphérique, d'assainissement des eaux urbaines ou de protection de la nature) se sont fixées pour objectifs de protéger les êtres humains, la faune et les plantes ainsi que leurs biocénoses contre des atteintes dangereuses ou nuisibles, en réduisant ces dernières à un niveau considéré comme supportable. En un mot, elles sont conçues comme des moyens de lutter contre les immissions ${ }^{2}$ par l'imposition de réductions des émissions.

$\mathrm{Si}$, en Suisse, ces politiques de protection ont incontestablement porté leurs fruits, notamment en ce qui concerne la protection des eaux et de l'air ou le traitement des déchets, leur succès demeure sensiblement moindre dans le domaine de la protection des sols, de la nature et du paysage ou encore de la biodiversité (OCDE 2007). De plus, il n'existe guère de signes que ces politiques aient contribué à rendre plus durable l'exploitation des ressources naturelles, renouvelables ou non. Ces constats obligent les observateurs à reconsidérer en profondeur la conception de toutes les politiques publiques à incidences environnementales, qu'elles relèvent de la protection ou de l'exploitation - y compris «écologiquement propre» - des ressources

${ }^{2}$ L'immission caractérise la concentration des polluants dans l'air ambiant. C'est le stade final du cycle de la pollution atmosphérique qui concerne la qualité de l'air après concentration des polluants primaires (venus de l'émission) et des polluants secondaires créés après transformation des polluants primaires. Le terme immission provient du latin: immittere $=$ envoyer dans, tandis qu'émission vient d' emittere $=$ envoyer à partir de. 
naturelles (par exemple les politiques d'aménagement du territoire, infrastructurelle, énergétique ou agricole). En effet, il s'agit de dépasser le véritable paradoxe des politiques environnementales classiques selon lequel « moins il y a d'émissions, plus on pourra admettre l'exploitation».

Plus encore, il apparaît impératif de prendre en compte l'ensemble des règles institutionnelles codifiant les rapports sociaux qui sous-tendent l'usage des ressources naturelles. Au-delà des politiques publiques - qui ont retenu l'attention soutenue des politologues - il s'agit aussi d'intégrer explicitement, dans l'analyse des «configurations institutionnelles » qui influencent le DD, les droits de propriété octroyés à certains usagers des ressources naturelles - droits qui sont considérés comme la règle institutionnelle déterminante par la plupart des économistes des ressources.

Cet article propose une approche théorique - le cadre des régimes institutionnels de ressources (RIR) - qui vise à identifier les divers «modalités de régulation» des usages concurrents des ressources naturelles. Notre démarche consiste à analyser l'ensemble des régulations, essentiellement formelles, régissant les usages des ressources naturelles de façon à pouvoir mettre en évidence les mécanismes expliquant leur dégradation ou au contraire (quoique plus rarement) les conditions de leur gestion durable. Il nous semble opportun de confronter ce cadre analytique, qui a été développé en combinant les approches de l'économie institutionnelle des ressources et de l'analyse des politiques publiques, au corpus de la théorie française de la régulation (TR, telle que formalisée notamment par M. Aglietta, R. Boyer et A. Lipietz). Inversement, nous avons la conviction que la TR peut profiter de certains éléments développés par l'approche des RIR, ceci notamment dans la perspective du présent numéro de la Revue de la Régulation consacré aux relations entre TR et DD.

Développée en France depuis les années 1970, la TR intègre l'analyse de la société civile et de l'action de l'État à l'économie politique, afin de mettre en évidence les mécanismes qui expliquent la pérennité du capitalisme en dépit des contradictions qui lui sont inhérentes (Jessop 1997). Ce faisant, la TR s'intéresse à la question du changement institutionnel à la compréhension duquel R. Boyer (2003) s'est plus particulièrement attelé en s'interrogeant sur l'apport des analyses historiques comparatives menées par certains politologues travaillant dans la perspective de la sociologie historique de l'Etat (Thelen 2003). Cependant, en se concentrant sur les interconnexions entre les formes institutionnelles et la stabilisation (temporaire) des économies capitalistes, la TR ne traite pas spécifiquement des usages sociaux et économiques des facteurs de production essentiels que représentent les ressources naturelles, dont la durabilité de la gestion constitue cependant une condition centrale de la reproduction du système capitaliste. C'est précisément l'objectif de notre contribution que de proposer un pont, sous la forme du cadre conceptuel des RIR, entre la TR et la régulation des usages (durables) des ressources naturelles.

Cet article poursuit ainsi un double objectif : premièrement, présenter le cadre d'analyse des RIR qui permet d'analyser, sur le long terme et à différentes 
échelles, les relations existant ou non entre les règles institutionnelles, les usages rivaux des ressources et le DD; et, secondement, tester la compatibilité entre le cadre des RIR et la TR, soit les complémentarités possibles entre ces deux approches dans la perspective d'une meilleure compréhension des rapports entre les changements de configurations institutionnelles et la durabilité.

Nous opérons ce test, d'une part, en discutant à partir du point de vue des RIR certaines des propositions faites par R. Boyer dans son article appelant à un renouvellement du programme de recherche régulationniste (Boyer 2003 : 197$199)^{3}$. Ce qui nous incite à identifier les points de convergence et les complémentarités théoriques et conceptuelles entre les deux approches, de même que les apports spécifiques des RIR à la TR. D'autre part, nous formulons une hypothèse générale concernant les effets, en termes de durabilité, des différents types de RIR qui découlent des changements institutionnels identifiés par R. Boyer.

Cet article s'articule en trois parties. La première montre comment la combinaison de l'économie institutionnelle des ressources naturelles, qui insiste sur l'importance des droits de propriété, et de l'analyse des politiques publiques, qui identifie les mécanismes qui sous-tendent les interventions sectorielles de l'État, permet de rassembler les variables explicatives les plus importantes pour une compréhension fine de la régulation des usages des ressources naturelles. Ces variables sont ensuite ordonnées sous la forme du cadre d'analyse des RIR permettant une approche systématique de la régulation des ressources. La deuxième partie décrit la trajectoire historique de la régulation d'une ressource particulière - le sol - dans le contexte helvétique de 1870 à nos jours. Cette étude de cas exemplifie l'utilisation du cadre des RIR. Finalement, la troisième partie développe plusieurs pistes de réflexion sur la compatibilité entre RIR et TR.

\section{Les régimes institutionnels de ressource (RIR)}

Le cadre théorique des régimes institutionnels de ressources (RIR) prend en compte, de manière conjointe, les systèmes de droits de propriété (SDP) sur

\footnotetext{
${ }^{3}$ Les propositions de R. Boyer pour une «troisième étape de la théorie de la régulation » sont les suivantes: (1) la viabilité d'une institution dépend de sa capacité générative et de ses potentialités d'adaptation, et non pas de la permanence de ses traits; (2) les théories de la "dépendance au sentier» n'éclairent que très partiellement la variété et la spécificité de l'évolution des institutions, car elles négligent leur endométabolisme; (3) les approches historiques comparatives permettent de révéler la variété des formes d'évolution institutionnelle (conversion, sédimentation et recombinaison); (4) la viabilité d'une configuration institutionnelle ne résulte pas nécessairement de l'intensité du couplage entre ses éléments constituants, (5); il est nécessaire de distinguer et d'affiner conceptuellement les différents types de règles institutionnelles (constitution, organisation, règle (loi, règlement), routine, convention ou habitus) ; et, (6) pour monter en généralité, il faut opérer une mise en parallèle de l'analyse historique comparative et de la TR, sous l'angle de la conception des institutions et de l'explicitation des facteurs de changements.
} 
une ressource naturelle et l'ensemble des politiques publiques (PP) qui en régulent l'exploitation et la protection. Il intègre les dimensions essentielles à l'analyse que sont les ressources naturelles, les acteurs usagers et les règles institutionnelles qui influencent le comportement des usagers (Knoepfel et al. 2007). Ces trois dimensions sont aussi constitutives de l'économie institutionnelle des ressources, dont par exemple l'abondante littérature sur les ressources exploitées en commun ou «common pool resources» (Ostrom 1990). Le postulat central de l'approche par les RIR affirme que les droits sur la ressource et les politiques publiques doivent être considérés simultanément de façon à comprendre les effets des régulations étatiques sur les usages sociaux des ressources. Se complétant l'une l'autre, ces deux dimensions régulatrices traduisent la volonté collective de protéger, garantir ou interdire tel ou tel usage à un moment donné dans le temps et l'espace. Le cadre analytique des RIR permet d'identifier les pratiques de gestion en vigueur. Il se base sur les quatre propositions suivantes :

(1) Toute définition d'une ressource naturelle est contingente. Elle dépend des perceptions socioculturelles et des comportements socio-économiques d'une collectivité. Une ressource naturelle est reconnue collectivement puis politiquement en tant que telle, dès lors que ses usagers reconnaissent ses biens et services dérivés comme contribuant à satisfaire leur besoins matériels et immatériels. Corollairement, la définition d'une ressource naturelle varie de manière significative dans le temps (entre générations) et dans l'espace (entre régions), en fonction des différentes attentes sociales - c'est-à-dire de la demande de certains biens et services - qui y sont articulées.

(2) Les utilisateurs d'une ressource peuvent obtenir des droits d'usage concrétisés par un accès privilégié à un "flux de bénéfices» fournis par la ressource (Bromley 1991) - par l'acquisition de droits de propriété et/ou par les actes de mise œuvre d'une politique publique autorisant l'usage de certains biens et services. Par exemple, un permis de construire contient un droit d'usage implicite d'utiliser les capacités d'absorption de l'atmosphère lors du chauffage du bâtiment (émission de $\mathrm{CO}_{2}$ ). Il en va de même pour le permis de circulation et la plaque minéralogique d'une automobile qui, de fait, confèrent également un droit illimité à polluer à leur détenteur.

(3) Un droit d'usage est considéré en tant que tel si des institutions (y compris informelles) protègent son détenteur contre d'autres usagers potentiellement intéressés par le même «flux de bénéfices ». Historiquement, la garantie (de la stabilité) des règles régulant les relations entre les citoyens par rapport aux biens en leur possession représente l'un des premiers domaines d'action des États modernes. Cet effort a conduit au développement du droit privé qui, en précisant les droits (et les devoirs) des citoyens les uns envers les autres, a également codifié les attributs de la propriété. Comme O. Steiger (2006) le souligne, le courant dominant dans la science économique utilise le terme de «droit de propriété » pour qualifier de simples «règles possessionnelles » faisant référence à l'usage ou au contrôle matériel (non formalisé dans le droit) des biens et des ressources. Nous nous référons toutefois ici aux droits de 
propriété en tant que revendications inscrites dans et protégées par le droit. Un droit de propriété dans le sens du Code civil transforme les règles possessionnelles en des droits d'appropriation exclusive des «flux de bénéfices» régis par la loi. Dans les États de droit, cela signifie qu'une analyse précise des bases légales du systèmes de droits de propriété en vigueur est indispensable à la compréhension des modalités de régulation des usages sociaux des ressources.

(4) Tout RIR a une influence directe sur l'état de la ressource au travers de la réglementation qualitative et quantitative des usages que les détenteurs de droits peuvent faire de la ressource. Par conséquent, le cadre des RIR postule une relation causale entre le type de régime (ou de la "configuration institutionnelle » pour adopter les termes de la TR) et la durabilité de la ressource.

Le concept de RIR a été jusqu'à présent conçu essentiellement dans une perspective heuristique. Il a pris naissance et a évolué au cours de nos tentatives de développer un système d'analyse des conflits autour de l'usage des ressources dans un contexte social, économique, juridique et politique particulier, celui de la Suisse (Kissling et Varone, 2000 ; Knoepfel et al. 2001, 2003 ; Rodewald et Knoepfel 2005). Sa mise en œuvre dans des projets de recherche européens (Bressers et Kuks, 2004; Kissling-Näf et Kuks, 2004) a cependant démontré la pertinence de sa transposition dans d'autres contextes institutionnels (dont par exemple la France, l'Espagne, l'Italie, la Belgique ou les Pays-Bas).

\subsection{Politiques publiques vs droits de propriété}

Dans le droit suisse, comme dans de nombreux systèmes juridiques romanogermaniques, une distinction est généralement faite entre le droit privé, codifié dans un Code civil et différentes lois complémentaires (par exemple celles concernant la propriété intellectuelle), et le droit public, rassemblé dans un corpus de lois. Le droit privé codifie les relations entre les personnes juridiques (c'est-à-dire des individus, des firmes, des ONGs, etc.), tel que le droit des contrats, de voisinage, de propriété, de succession, etc. Il se distingue du droit public qui traite des relations entre les personnes juridiques et l'État, et qui, par le biais des politiques de régulation, détermine la frontière entre l'intérêt public et l'intérêt privé.

Les droits de propriété sont la manifestation juridique de la garantie d'accès à un flux de bénéfice dans le contexte d'un ordre politique et social particulier. Ils s'appliquent uniquement à des éléments du monde empirique juridiquement reconnus comme des "choses", soit des objets matériellement saisissables on maîtrisables. Dans ce sens, un élément qui n'a pas de réalité matérielle ne peut être l'objet d'une relation de propriété. Par conséquent, dans l'ordre juridique en vigueur, il ne peut y avoir de droits de propriété sur des ressources tels que le paysage ou l'air, ceci même s'il existe des mécanismes d'appropriation en quelque sorte «indirects», issus principalement des politiques publiques. On peut généralement distinguer trois « niveaux » de droits de propriété :

(1) Les droits de propriété formelle définissent qui est le propriétaire d'une (portion

de) ressource naturelle et quels sont les droits fondamentaux du propriétaire 
sur cette ressource. Ils doivent être distingués des droits d'usage attribués par des politiques publiques, dans la mesure où ils sont fondés dans le Code civil (CC) ce qui les rend beaucoup plus stables et persistants ${ }^{4}$ et les protègent en principe mieux contre les atteintes portées par des individus, des organisations tierces ou l'Etat. Alors que les atteintes portées aux droits de propriété et d'usage fondés sur le CC impliquent souvent une compensation ou une indemnisation, le retrait ou la modification de droits (d'usage, d'accès ou d'appropriation) basés sur les politiques publiques (par exemple licences d'émission de substances polluantes, droit de circuler conféré par les plaques d'immatriculation, etc.) sont généralement plus faciles et donnent moins fréquemment droit à une indemnisation. Notons encore que, outre les restrictions fréquentes par les politiques publiques des droits de propriété fondés sur le CC, ce dernier ouvre lui-même la voie à de nombreuses limitations au contenu des droits de propriété formelle, par l'intermédiaire notamment des servitudes et du droit du voisinage (cf. Kirat 2005).

(2) Les droits de disposition fixent les conditions sous lesquelles un propriétaire formel peut disposer librement ou non (d'une partie) de la ressource. Ils concernent les modalités de transfert des droits de propriété formelle, soit le droit de vendre, de louer, d'hypothéquer, de transmettre en héritage, ou de donner la chose possédée. Les droits de disposition découlent du droit de propriété formelle dans le sens où seul le propriétaire d'une chose peut décider du transfert de la propriété de la chose possédée. Néanmoins, il existe des instruments juridiques qui limitent la capacité du propriétaire à disposer de sa propriété, tels que le droit d'expropriation, les interdictions de diviser les terrains agricoles ou, pour les fondations, l'interdiction de dilapider leur patrimoine.

(3) Les droits d'usage (y compris les droits de gestion et de prélèvement) définissent qui peut faire quel usage de quelle quantité de la ressource sous la forme de quels biens et services dérivés de celle-ci. La définition de ces droits d'usage résulte de la combinaison de normes découlant des droits privé et public. Alors que le droit privé établit dans le CC la base de la propriété absolue, le droit public tempère ce droit absolu en imposant des restrictions d'usages au travers des politiques publiques (cf. Art. 641 du Code civil suisse). Les droits d'usage sont plus spécifiques que les titres de propriété formelle dans la mesure où ils ne concernent généralement qu'un seul bien ou service dérivé de la ressource naturelle. De plus, tous les droits d'usage ne découlent pas directement de droits de propriété formelle : ils peuvent également dériver, comme évoqué plus haut, de politiques publiques qui créent des droits d'usage et les attribuent à des bénéficiaires formellement non propriétaires.

La définition des droits d'accès, en tant que catégorie spécifique de droits d'usage, s'inscrit dans la même logique. Les propriétaires d'une parcelle ont le droit d'exclure les intrus. Toutefois, dans certains cas, le droit d'exclusion est

${ }^{4}$ En Suisse, le CC n'a pas subi de changements fondamentaux depuis son introduction en 1912. 
limité par le droit public (par exemple, l'«allemansrätt» de la Constitution suédoise, littéralement le "droit de tout un chacun», garantit le principe du libre accès public aux pâturages, tout comme l'article 699 du Code civil suisse) ou par le droit privé (par exemple, les droits de passage pour les voisins ou pour le public inscrits au registre foncier). Un accès minimal est garanti, dans de nombreux pays, par des droits constitutionnels comme la liberté de mouvement, du moins en ce qui concerne les biens publics (par exemple les rues, les places ou les cours d'eau).

\subsection{Modalités de régulation}

La figure 1 représente graphiquement les différents éléments constitutifs d'un RIR et, en mettant en évidence les relations qui les lient les uns aux autres, décrit les différentes modalités de régulation des usages d'une ressource naturelle pouvant résulter d'une telle configuration institutionnelle.

Fig. 1 - Modalités de régulation au sein d'un RIR

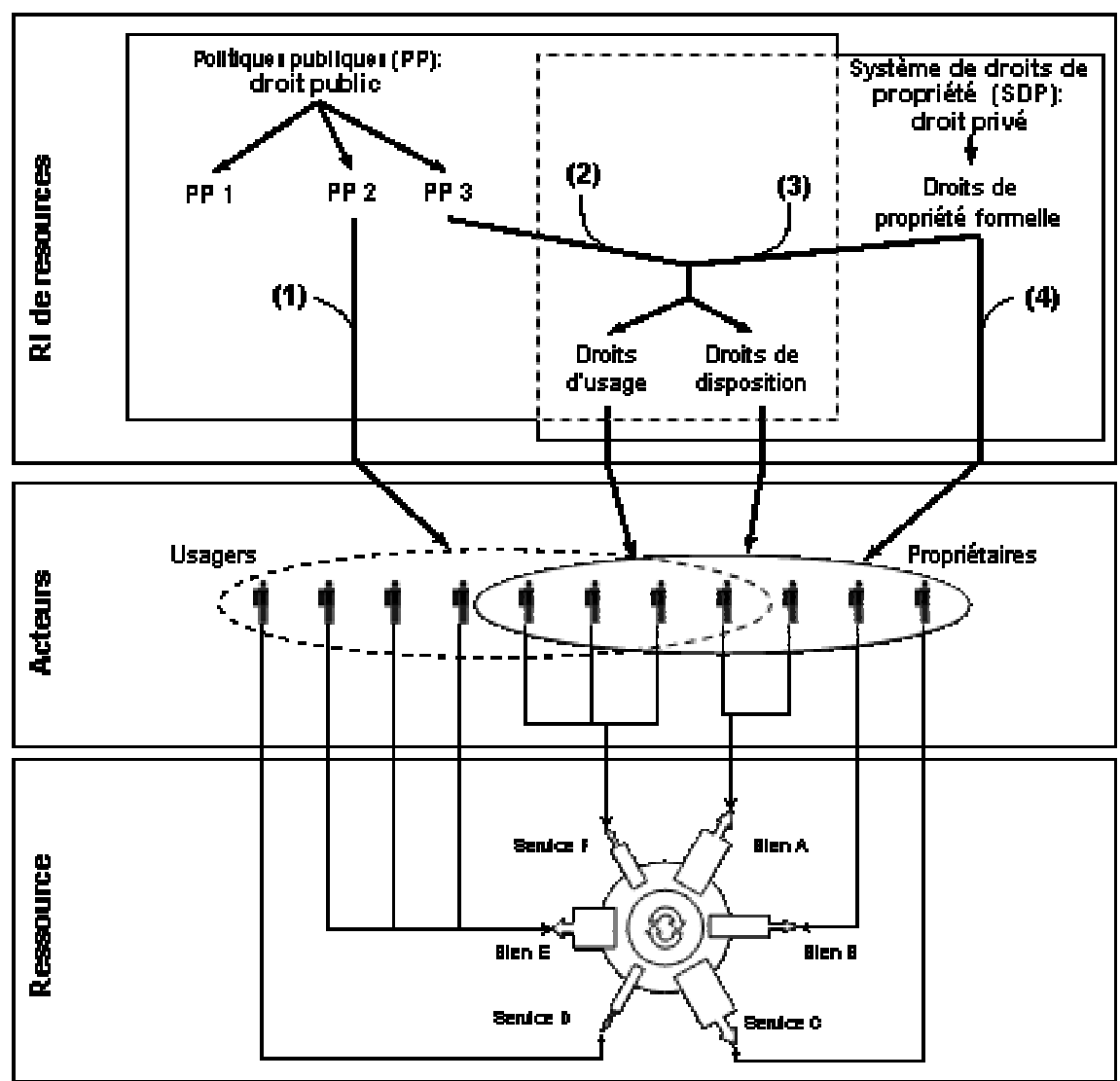

Un RIR est composé, d'une part, d'un système de droits de propriété (SDP, droit privé) et, d'autre part, de l'ensemble des politiques publiques (PP, droit public) régulant les différents usages (rivaux) faits par les différents groupes d'utilisateurs de la ressource. Ce faisant, il influence significativement la durabilité de l'état de la ressource. Une situation d'usage durable implique que 
le capital de la ressource ne soit pas affecté par la somme des différents usages simultanés (biens et services consommés) de celle-ci.

Il est possible de distinguer quatre modalités de régulation des usages d'une ressource issus d'un RIR :

(1) Le premier mode est celui de la régulation par le biais de politiques publiques (PP) n'ayant pas d'effets sur le contenu des droits de propriété (SDP). Ce mode de régulation implique la mise en œuvre d'instruments incitatifs comme par exemple des campagnes d'information, des subsides ou des allègements fiscaux pour encourager un comportement particulier (par exemple des subventions pour soutenir l'agriculture biologique).

(2) Le deuxième mode opère également au travers des $P P$, mais cette fois-ci avec un impact sur la valeur et le contenu des droits de propriété. Il correspond au recours, par l'Etat, à des instruments de PP ayant un impact sur les droits de disposition et/ou sur les droits d'usage par le biais d'une redéfinition du contenu substantiel de ces droits, par exemple sous la forme de restrictions en matière de construction, de limitation des droits d'émission de polluants atmosphériques, de prélèvements (de bois, de plantes rares ou de gibier), ou encore des droits d'accès (aux rives des lacs, aux forêts ou aux biotopes fragiles). L'un des enjeux majeurs de ce mode de régulation consiste à définir les conditions pour l'obtention, par les propriétaires lésés, d'indemnisations ou de compensations.

(3) La redéfinition de l'institution même du droit de propriété, opérée principalement au travers (d'une révision) du Code civil, constitue un troisième mode de régulation. Une telle intervention implique une modification de la portée substantielle des droits de propriété, notamment du contenu des droits de disposition et d'usage. En Suisse, l'exemple le plus important d'un tel changement consiste dans l'introduction du Code civil suisse qui a créé une définition unifiée du droit de propriété, au niveau fédéral, et a aboli définitivement les anciens droits d'usage et de disposition cantonaux (par exemple les régimes de propriété commune) en particulier dans le domaine du droit foncier. Un exemple plus récent est celui de l'introduction en 1965, dans le Code civil, de la propriété par étage.

(4) Le quatrième mode de régulation procède par une redéfinition de la structure de distribution des droits de propriété. Celle-ci peut consister en une intervention aussi radicale que la privatisation ou la nationalisation (de terres, de cours d'eau, de forêt, etc.) pour toutes sortes de raisons (l'amélioration de l'efficience économique, la sécurité de l'approvisionnement, l'effort pour contrecarrer la spéculation, etc.) ou une intervention plus ponctuelle et limitée consistant en une expropriation formelle (par exemple pour la mise en œuvre de projets d'infrastructures publiques).

\section{3. Étendue et cohérence des RIR}

Les RIR peuvent être caractérisés et catégorisés sur la base de deux dimensions analytiques que sont l'étendue et la cohérence. L'étendue d'un RIR fait référence au nombre de biens et services régulés par le régime à un temps donné. Le critère 
de la cohérence renvoie au contenu et à l'articulation entre elles des différentes régulations de droit public (PP) et privé (SDP) constitutives du régime. Plus ces régulations sont nombreuses et diverses (étendue élevée), plus le risque d'incohérence entre elles est élevé. Ces incohérences peuvent concerner les régulations issues du SDP, des PP ou encore de la rencontre entre les deux :

(1) La cohérence interne du SDP fait référence au degré de clarté de la définition des droits de propriété et des droits de disposition et d'usage qui en résultent. Par exemple, des incohérences dans le SDP peuvent dériver de situations où il y a plus de prétendants autorisés pour une seule ressource ou un seul bien et service qu'il n'y a d'unités de ressource effectivement disponibles (par exemple le pompage libre et non régulé de la nappe phréatique par des forages privés).

(2) La cohérence interne des PP décrit surtout le degré de coordination entre les politiques d'exploitation et celles de protection des ressources naturelles. Dans les années 1990, cette coordination a souvent été faible et parfois inexistante. Des PP incohérentes produisent généralement des régulations d'usage qui sont incompatibles les unes avec les autres (cf. les contradictions entre la politique d'économie d'énergie et celle de libéralisation du marché de l'électricité, entre le soutien à une agriculture intensive et la protection des sols, des eaux et de la biodiversité, ou encore entre la contradiction entre la politique de concurrence fiscale entre les territoires et la politique d'aménagement du territoire) ${ }^{5}$.

(3) La cohérence externe décrit la manière dont les deux composants d'un RIR sont articulés. Elle dépend en particulier de la correspondance entre le groupecible d'une PP et le groupe des détenteurs de droits tels que définis dans la pratique par le SDP. Cette correspondance fait défaut lorsque les PP désignent comme groupe-cible des acteurs qui n'ont pas de droits d'usage ou dont les actions n'ont pas de véritables effets sur l'état de la ressource (par exemple l'obligation faite aux communes de respecter des débits minimaux dans des cours d'eau dont elles ont perdu le contrôle suite à l'octroi de concessions à des compagnies hydroélectriques). D'autres incohérences externes existent dans le cas relativement répandu où une PP n'arrive pas à restreindre les droits d'usage des propriétaires d'une ressource. À titre d'exemple, citons la capacité qu'ont les propriétaires fonciers à résister à la planification de l'aménagement du territoire et à l'éventuel déclassement de leur terrain dans une zone non constructible. L'incohérence externe d'un RIR se manifeste souvent par une grande conflictualité et la production d'une quantité élevée de jurisprudence par les tribunaux (cf. Kirat et Melot 2006), ceci afin de coordonner les deux composantes du régime (Nahrath, 2005). Tout comme les décisions des tribunaux, certains arrangements locaux informels peuvent également restaurer la cohérence au sein d'un RIR victime de complexité croissante.

\footnotetext{
${ }^{5}$ Des contradictions à l'intérieur d'une politique publique (par exemple entre la définition du problème, la logique d'action, les instruments d'intervention, l'arrangement politicoadministratif de mise en œuvre, etc.) existent également.
} 


\subsection{Typologie des RIR}

Une des contributions fondamentales du cadre analytique des RIR est sa capacité à décrire théoriquement et empiriquement les différents régimes de régulation. Une typologie simple distingue quatre types différents de RIR selon leur étendue et leur cohérence (Fig. 2).

Fig. 2 - Typologie des RIR.

\begin{tabular}{|c|c|c|c|}
\hline \multicolumn{2}{|c|}{ Types de RIR } & \multicolumn{2}{c|}{ Cohérence } \\
\cline { 3 - 4 } \multicolumn{2}{|c|}{} & Faible & Élevée \\
\hline \multirow{2}{*}{ Étendue } & Faible & RIR inexistant & RIR simple \\
\cline { 2 - 4 } & Élevée & RIR complexe & RIR intégré \\
\hline
\end{tabular}

(1) On parle ainsi de régime inexistant dans le cas où aucun droit de propriété, ni aucune disposition d'une PP ne régule l'usage des biens et service d'une ressource. Pareil cas est observé par exemple lorsque le besoin de régulation d'une ressource n'a pas encore été reconnu politiquement malgré le fait que la ressource est l'objet d'une variété d'usages (par exemple la ressource génétique jusqu'à très récemment).

(2) Nous désignons comme un régime simple les situations où un nombre limité de biens et services est régulé de manière cohérente, cette cohérence étant facilitée par le faible nombre de régulations. Une telle situation résulte fréquemment d'une première tentative de régulation des principaux usages rivaux d'une ressource. La raison d'être de tels RIR n'est souvent pas de protéger la ressource en tant que telle, mais plutôt de garantir son accès à ses principaux exploitants afin d'assurer une possibilité d'usage sur le long terme ou l'amortissement des installations d'exploitation construites aux frais de l'exploitant (par exemple des concessions hydroélectriques).

(3) Un régime complexe se caractérise par le fait que la majorité des biens et services utilisés est régulée, mais de manière partiellement incohérente. Cette situation, qui prévaut pour la plupart des régimes de ressource à la fin du $20^{\circ}$ siècle en Suisse, est due au développement non coordonné de PP d'exploitation et de protection. Contrairement aux régimes simples, les RIR complexes sont essentiellement issus d'une mobilisation politique visant à trouver des solutions à des problèmes résultant de rivalités d'usage mettant en péril la capacité de reproduction de ressources naturelles. Ces régimes impliquent donc des tentatives, plus ou moins contraignantes, de formuler des limitations d'émission par secteur d'activité, au minimum au niveau des biens et services pris séparément (par exemple en imposant une réduction des émissions de polluants atmosphériques aux industries émettrices). À l'incohérence entre PP s'ajoute encore fréquemment une incohérence entre PP 
et SDP, les limitations des droits d'usage par les PP entrant en contradiction avec la garantie des droits de propriété.

(4) Finalement, une situation où tous les biens et services produits par une ressource sont régulés de manière cohérente est qualifiée de régime intégré. De tels régimes restent rares au début du $21^{\mathrm{e}}$ siècle. Ils existent lorsqu'une ressource est largement en propriété publique, par exemple les forêts, ou sous le contrôle d'un acteur collectif puissant, par exemple des institutions d'autogestion collective de ressources, telles que décrites par Ostrom (1990), comme les corporations, les organisations de conservations de la nature, les trusts anglo-saxons pour la nature. En Suisse, des exemples peuvent être trouvés dans le domaine de la forêt (Bisang et Schenkel, 2003: 198 ss.), de paysages spécifiques (Rodewald et Knoepfel, 2005 : 347 ss.; Gerber, 2006 : 333) et de gestion de l'eau par sous-bassins versants (Varone et al., 2002).

\subsection{Hypothèses de recherche}

Deux hypothèses centrales sur les relations causales entre la régulation institutionnelle et l'usage durable des ressources naturelles peuvent être dérivées du cadre des RIR (Fig. 3).

Fig. 3 - Hypothèses selon le cadre des RIR

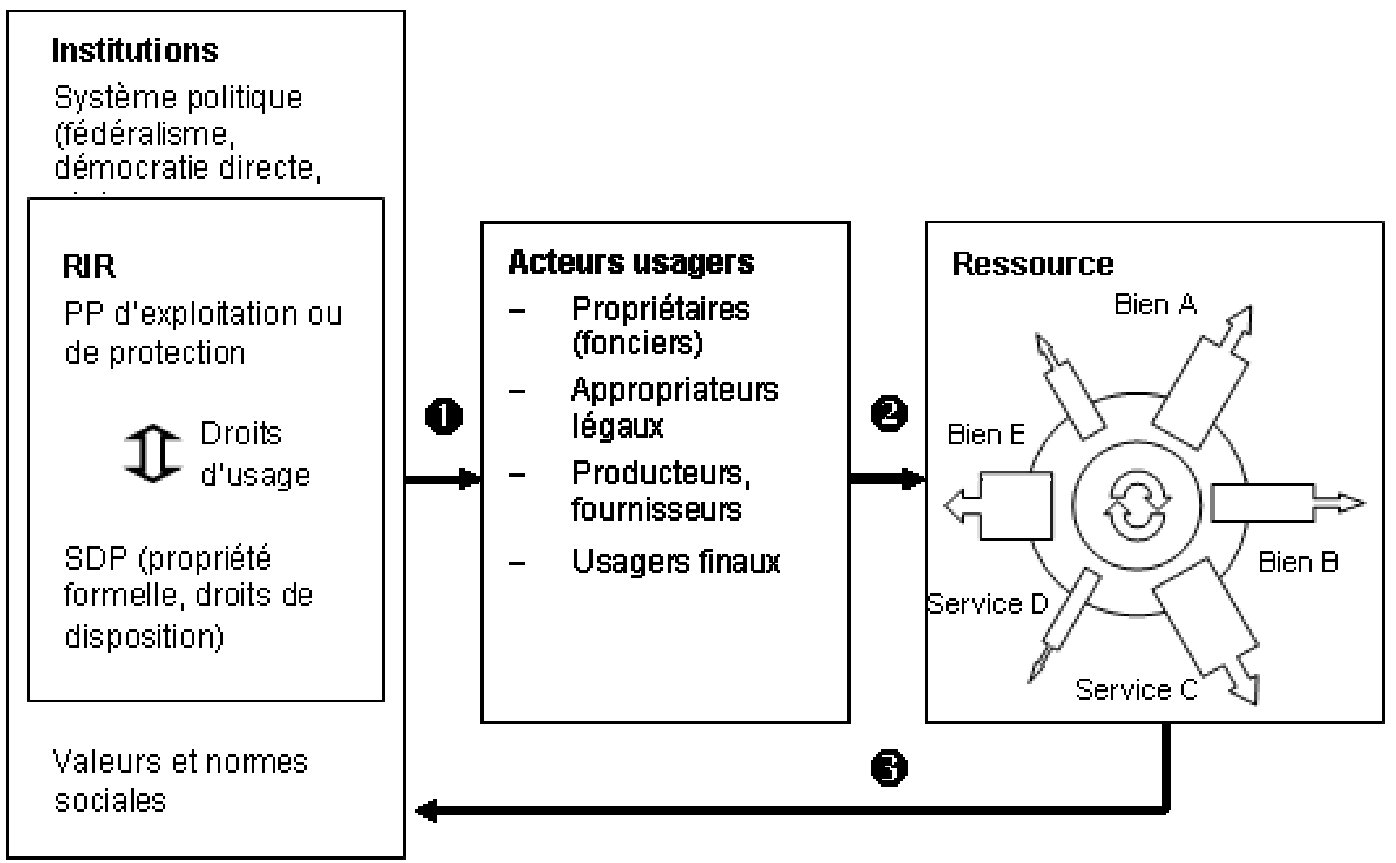

La première hypothèse (flèches 1 et 2 de la Fig. 3) se base sur la typologie des régimes présentée dans la Fig. 2. Elle présuppose l'existence d'une relation causale entre le type de régime (c'est-à-dire, son étendue et sa cohérence) et la durabilité des usages d'une ressource et donc sa capacité de reproduction. Par conséquent, plus un régime est proche de l'intégration, plus grande est la 
chance qu'il crée des conditions d'usage durable de la ressource. Inversement, plus la cohérence et l'étendue du régime sont faibles, plus grands sont les risques de surexploitation. Plus concrètement, la première hypothèse peut être divisée en deux hypothèses secondaires liées aux dimensions de (1.1) l'étendue et de (1.2) la cohérence. L'hypothèse 1.1 est basée sur l'idée qu'un manque de régulation du comportement des usagers - en l'absence d'une description précise des droits d'usage par les PP ou le SDP - risque d'engendrer des comportements stratégiques qui peuvent conduire à la surexploitation des ressources. L'hypothèse 1.2 est basée sur l'idée que des lacunes ou des incohérences dans les PP ou dans le SDP (cohérence interne), ainsi que entre les deux composantes des RIR (cohérence externe), constituent une cause majeure de surexploitation.

La deuxième hypothèse centrale (flèche 3 dans la Fig. 3) a trait à la relation causale inverse qui explique les dynamiques historiques de développement des RIR, ainsi que les causes majeures de changement de régime. L'hypothèse stipule que plus la durabilité de la ressource est menacée, plus forte est la probabilité qu'on observe une augmentation de l'étendue du régime (nouvelle régulation pour de nouveaux usages) ou une amélioration de la cohérence (en introduisant de nouveaux mécanismes plus contraignants afin de coordonner les actions des différents acteurs).

Sans pouvoir développer ici plus avant le cadre théorique du RIR, nous illustrons ses modalités d'application empirique en présentant une étude de cas portant sur la régulation du sol en Suisse de 1870 à nos jours.

\section{Trajectoire historique de la régulation du sol en Suisse (1870-2000)}

Afin d'illustrer les potentialités analytiques du concept de RIR dans une perspective historique, nous reconstituons successivement le développement historique des droits de propriété ainsi que des politiques publiques qui en régulent les usages, ceci afin d'identifier les principaux changements de régimes durant cette période, ainsi que les principales caractéristiques du régime actuel susceptibles d'expliquer la (non) durabilité des usages contemporains du sol. On peut identifier empiriquement un nombre très important d'usages différents du sol, le plus souvent concurrents car exclusifs, qui constituent autant de modalités de captation des biens et services (B\&S) produits par cette ressource (cf. Nahrath 2003 pour une liste de ces différents usages). Comme l'ont clairement montré nos analyses historiques (Nahrath 2003, 2005), l'augmentation de l'intensité des usages ainsi que l'hétérogénéisation des groupes d'usagers de la ressource au cours du temps ont nécessité la mise en place de régulations aussi bien dans le registre des droits de propriété (définition des principales dispositions du droit foncier), que dans celui des politiques publiques (politiques à incidences spatiales et politiques environnementales et d'aménagement du territoire). Dans les sections qui suivent (3.1 à 3.3), nous reconstituons successivement les différentes étapes de 
mise en place de ces régulations, ceci afin, dans un deuxième temps, de décrire à travers leur combinaison la dynamique de développement des régimes institutionnels du sol sur la période allant de la fin du $19^{\text {ème }}$ au début du $21^{\text {ème }}$ siècle.

\subsection{L'évolution des droits de propriété}

Notre analyse historique a permis d'identifier six grandes étapes dans l'évolution de la définition de la propriété foncière en Suisse durant la période considérée. La première période (1850-1912) correspond à la mise en place de compétences fédérales en matière d'expropriation foncière pour des raisons d'utilité publique. Les dispositions constitutionnelles adoptées en 1874 et 1897 se limitent à octroyer à la Confédération un instrument d'accès au sol se trouvant en mains privées de manière à permettre la réalisation d'infrastructures d'utilité publique dans les domaines des chemins de fer, de la poste et du télégraphe ou encore de la police des endiguements, des constructions hydrauliques et des forêts. Ces compétences fédérales sont attribuées dans un contexte où il n'existe pas de définition homogène de la propriété au niveau national (absence d'un code civil fédéral), diverses formes de propriété commune (consortages, bourgeoisies, corporations, etc.) continuant de coexister avec la propriété privée du sol dans les codes civils cantonaux.

La deuxième période (1912-1940) est marquée par la mise en place des fondements de l'ordre juridique libéral régissant, aujourd'hui encore, les relations de propriété. Les deux composants principaux de cette période sont le Code civil suisse (CC) qui entre en vigueur en 1912, et la nouvelle loi fédérale sur l'expropriation de 1930. Le CC consacre la propriété privée comme la seule forme juridiquement reconnue de propriété. Cette homogénéisation de la définition de la propriété a revêtu une importance historique centrale en conférant aux propriétaires - au nom du principe d'accession (art. 641, 655, 667 CC) - la «toute-puissance » sur les ressources possédées. Une lecture plus fine du Code civil permet cependant de voir que cette toute-puissance trouve déjà un certain nombre de limitations dans le droit privé lui-même ${ }^{6}$. Si le CC définit bien le principe de la propriété, il n'en définit cependant pas complètement, loin s'en faut, le contenu; l'alinéa 1 de l'article 641 stipule en effet que "le propriétaire d'une chose a le droit d'en disposer librement, dans les limites de la loi $\gg^{7}$ et laisse donc la porte ouverte aux interventions limitatives du contenu de la propriété par l'intermédiaire des politiques publiques (droit public).

Dans une troisième étape (1940-1955), on observe une focalisation des régulations sur les droits de disposition des propriétaires des terres agricoles

\footnotetext{
${ }^{6}$ Les quatre limitations les plus importantes sont le principe du libre accès aux forêts et aux pâturages et le droit de cueillette (art. 699); les rapports de voisinage (art. 684-698) et notamment le droit de passage (art. 694-696); les limitations du transfert des titres de propriété en matière de droit foncier rural en vue de la lutte contre la spéculation sur les terres agricoles (art. 621); ainsi que les servitudes foncières (art. 730-744) et le droit de superficie (art. 675 et 779).

${ }^{7}$ C'est nous qui soulignons.
} 
par l'intermédiaire de trois lois fédérales concernant la propriété foncière rurale (entre 1940 et 1951) et participant toutes d'une même tentative de protéger ces terres contre la spéculation foncière. Elles limitent notamment la capacité des agriculteurs à hypothéquer leurs terres ou à les vendre à des non-agriculteurs. Cette logique d'intervention focalisée sur les usages agricoles du sol se trouve renforcée par la mise en place du régime de l'économie de guerre (plan Wahlen de 1938 à 1945) débouchant sur une obligation quasi généralisée de mise en culture des terres, y compris dans les zones urbanisées.

Face au succès très relatif des interventions sur les droits de disposition des propriétaires des terrains agricoles, une quatrième étape (1955-1969) consacre une nouvelle limitation des droits d'usage, de l'ensemble des propriétaires cette fois-ci, ceci notamment par l'intermédiaire des premières tentatives de limiter le droit de construire sur les portions du territoire communal les plus clairement inappropriées pour cet usage. La loi sur la protection des eaux (1955 et surtout 1971) est ainsi mobilisée comme instrument de limitation de l'octroi de permis de construire dans le cas des parcelles situées en dehors du plan des canalisations. Parallèlement à ces premières tentatives de délimitation indirecte de la zone à bâtir, la concentration de la propriété foncière dans les mains des grandes sociétés de capitaux devient un problème politique. Ainsi, de manière à faciliter l'accès des particuliers à la propriété du sol ou d'un bien immobilier, la propriété par étages est introduite en 1963 dans le Code civil. Finalement, la loi sur la protection de la nature et du paysage (LPN, 1966) contribue également, même si elle ne s'inscrit pas dans une logique proprement aménagiste, à limiter les droits d'usage des propriétaires fonciers possédant des parcelles ou des objets classés dans les inventaires fédéraux.

La cinquième période (1969-1983) est absolument centrale dans la trajectoire historique de la (re)définition de la propriété foncière en Suisse. Les articles constitutionnels $22^{\text {ter }}$ et $22^{\text {quater }}$ (Bodenrechtsartikels) de $1969^{8}$ constituent en effet la «clé de voûte » du compromis social et politique "échangeant» la garantie constitutionnelle de la propriété et le principe d'indemnisation en cas d'expropriation d'un côté, contre l'inscription du principe de l'aménagement du territoire dans la Constitution, et donc la promesse de la mise en place d'une politique fédérale en la matière de l'autre. Alors que les articles constitutionnels de 1969 représentent une intervention sur les droits de propriété formelle (la dernière en date), la loi sur la protection des eaux de 1971, l'arrêté fédéral urgent en matière d'aménagement du territoire (AFU) de mars 1972 et finalement la loi sur l'aménagement du territoire (LAT) de 1979 interviennent de manière très significative sur les droits d'usage des propriétaires fonciers, notamment au travers de la suppression généralisée du droit de construire en dehors de la zone à bâtir. Avec la législation sur l'aménagement du territoire, c'est donc l'ensemble des propriétaires fonciers et immobiliers (privés comme publics) qui voient leurs droits d'usage profondément redéfinis et, souvent, limités de manière drastique.

\footnotetext{
${ }^{8}$ Respectivement articles 26 et 75 dans la nouvelle Constitution de 1999.
} 
Durant la dernière période (1983-2007), l'intervention régulatrice se concentre sur les droits de disposition (restrictions en matière d'acquisition d'immeubles par des personnes domiciliées à l'étranger et renforcement de l'interdiction de l'achat de terrains agricoles par des non-agriculteurs à travers la nouvelle loi sur le droit foncier rural de 1991) ainsi que sur les droits d'usage au travers de la loi sur protection de l'environnement (1983, révisée en 1995) et l'ordonnance sur la protection qualitative des sols (1998). Les limitations des droits d'usage concernent tout d'abord l'agriculture (engrais) et l'entreposage des déchets, puis l'exploitation des gravières et l'imperméabilisation des sols (plan communal de l'évacuation des eaux) et, enfin, la conduite de chantiers (usages des véhicules, machines et outils) et les techniques d'exploitation agricole des sols (lutte contre la compaction et l'érosion).

En conclusion, l'évolution du système de droits de propriété en Suisse se caractérise par deux grands mouvements successifs. Dans un premier temps, on assiste à la mise en place d'une conception homogène - très libérale - de la propriété foncière sur l'ensemble du territoire. Dans un second temps, on observe une complexification du contenu substantiel de la propriété foncière résultant de la lutte fondamentale entre, d'une part, des tentatives de limitation progressive des droits de disposition et d'usage par les politiques publiques (sectorielles) porteuses de la défense de l'intérêt général et, d'autre part, les efforts de consolidation juridique de la garantie de la propriété privée.

Ainsi, la prépondérance de l'intervention sur la définition des droits de propriété formelle dans les deux premières périodes s'explique par la proximité de la fondation de l'ordre social et politique de la Suisse moderne à la fin du XIXe et au début du XXe, moments de formalisation et d'institutionnalisation de principes sociaux, économiques et politiques fondamentaux, tel que précisément celui de la propriété privée dans une société capitaliste. De même, le passage par la limitation des droits de disposition durant les années 1940 et 1950 et le développement relativement tardif de la politique d'aménagement du territoire s'expliquent par le risque politique que représente précisément la limitation des droits d'usage, c'est-à-dire toute atteinte portée à la propriété foncière. Il s'agit là d'une tendance récurrente de la stratégie de régulation étatique en Suisse (fondée en partie dans le principe de subsidiarité) que de choisir systématiquement les interventions ayant le moins d'impacts directs sur la propriété privée des citoyens et surtout des entreprises. L'histoire de la difficile mise en place d'une politique fédérale d'aménagement du territoire en est une illustration des plus claires. Il a dès lors fallu attendre que la pression objective des problèmes, de même que, surtout, leur perception sociale et politique par des groupes sociaux structurés ayant des intérêts et des droits à faire valoir (notamment les agriculteurs, les locataires et les protecteurs de l'environnement et du paysage), atteigne un certain niveau de visibilité, pour ne pas dire d'urgence, pour que la limitation des droits d'usage des propriétaires fonciers devienne politiquement possible. 


\subsection{L'évolution des politiques publiques}

L'histoire des politiques publiques intervenant dans la régulation de l'exploitation comme de la protection du sol durant les cent dernières années peut être quant à elle divisée en quatre périodes.

Depuis la création de l'État fédéral en 1848 jusqu'à la Deuxième Guerre mondiale (première période), le sol n'a fait l'objet d'aucune régulation directe significative par l'intermédiaire de politiques publiques fédérales.

Par la suite, une deuxième période (1940-1955) se caractérise par des mesures étatiques ayant pour objectif de lutter contre la spéculation foncière sur les terres agricoles. Il s'agit notamment de rendre très coûteuse la réaffectation à d'autres usages des terres agricoles subventionnées (par une obligation de rembourser les subventions obtenues). Cette logique d'action désigne les agriculteurs (endettés ou tentés de le devenir) comme le groupe-cible unique des PP. Les spéculateurs, les banques et les entrepreneurs ne constituent ainsi à cette époque que des groupes tiers indirectement affectés par les mesures prises. Durant la Deuxième Guerre mondiale, le plan Wahlen d'accroissement des surfaces cultivées représente quant à lui un exemple - certes temporaire de planification des usages du sol affectant une surface jamais atteinte jusqu'ici dans l'histoire du pays. Les terres agricoles sont donc les premières portions significatives du territoire national (à part les forêts) à avoir connu une intervention étatique - certes encore limitée et indirecte - visant à lier des usages du sol à des parcelles particulières en fonction de leurs caractéristiques et de leur localisation. Ainsi, contrairement à d'autres pays européens (par exemple en France ou en Allemagne), la politique d'aménagement du territoire se développe en Suisse, non pas à partir des zones urbaines (cf. Code de l'urbanisme), mais à partir de la zone agricole.

Le développement d'une troisième étape (1955-1971) découle de la croissance urbaine, du développement des activités industrielles, de la construction d'infrastructures urbaines, d'habitations ou de transports, notamment la route et le rail, qui contribuent à un accroissement significatif de la demande de terres constructibles dans les zones urbaines et périurbaines. L'absence de prescriptions strictes en matière de droit de la construction a pour effet un étalement et une dispersion des constructions, ainsi que le développement d'opérations spéculatives (notamment sur les terres agricoles périurbaines, les vignobles, les zones touristiques et les rives des lacs) ayant pour conséquence, dans un pays aussi exigu et densément peuplé, une hausse généralisée des prix des terrains (explosion des prix qui explique aujourd'hui encore le niveau très élevé des loyers, ainsi qu'un taux de propriétaires de logements ne s'élevant qu'à 30\%). En conséquence, il est décidé de protéger progressivement l'ensemble des surfaces non construites, en instituant une distinction de facto - à défaut de de jure, en l'absence de politique d'aménagement du territoire - entre zones constructibles et non-constructibles (cf. les lois de 1955 et 1971 sur la protection des eaux, de 1963 sur la propriété par étages, de 1965 sur l'encouragement à la construction de logements ou de 1966 sur la protection 
de la nature (LPN)). Par l'intermédiaire de ces différentes lois, on tente d'intervenir, d'une part, sur la délimitation de la zone à bâtir, sur les conditions d'accès des classes moyennes à la propriété immobilière ou encore sur la construction de logements sociaux et, d'autre part, sur la mise sous protection des espaces naturels encore vierges ainsi que des paysages naturels et culturels dignes de protection. Au total, l'utilisation détournée de différentes lois pour pallier l'absence de politique coordonnée d'aménagement du territoire, si elle est ingénieuse compte tenu des circonstances, n'en constitue pas moins une situation clairement insatisfaisante.

L'adoption en votation populaire en 1969 des articles constitutionnels (Bodenrechtsartikels) concernant l'aménagement du territoire et la garantie de la propriété foncière débouche sur une quatrième période (1972-1983) que l'arrêté fédéral urgent (AFU) du 17 mars 1972 en matière d'aménagement du territoire inaugure de manière fracassante. La logique de cet AFU consiste à mettre de manière préventive, soit jusqu'à l'entrée en vigueur de la future LAT (1979), sous protection provisoire l'ensemble des territoires non construits dont l'affectation actuelle ou probable risque de ne pas être conforme aux principes de la future LAT. Reprenant à son compte le principe de la protection des espaces naturels et du paysage tel que le conçoit la LPN (1966), cet AFU opère une sorte de conversion de la logique de protection par inventaires dans une logique de zonage conforme à la philosophie de l'aménagement du territoire, préfigurant ainsi partiellement les futures zones protégées et agricoles (c'est-àdire non constructibles) de la LAT.

L'objectif central de cette nouvelle politique fédérale d'aménagement du territoire est d'assurer une «utilisation judicieuse du sol » et une " occupation rationnelle du territoire» (art. 1 LAT). L'application de ces deux principes implique deux grandes opérations : la séparation stricte entre la zone à bâtir et les autres zones (agricole et protégée) ainsi qu'une meilleure utilisation des zones déjà bâties. Il s'agit ici, de fait, de la première régulation généralisée, explicite et directe $d u$ droit de construire sur l'ensemble des parcelles du territoire national. L'instrument du zonage attribue ainsi à chaque parcelle une identité administrative et une définition claire de ses usages possibles.

Les impacts financiers résultant de la mise en œuvre du zonage (multiplication du prix du sol par 100 ou 1.000 en cas de classement en zone à bâtir) ont provoqué d'énormes conflits entre autorités communales et propriétaires fonciers lors de la réalisation de la première génération des plans d'affectation communaux, les propriétaires désavantagés - ou carrément lésés en cas de retrait du droit à bâtir - ayant dans de nombreux cas recouru devant les tribunaux. Ces derniers constituent ainsi des acteurs importants de cette quatrième étape, puisqu'ils ont dû définir, dans le cadre d'une jurisprudence détaillée, les conditions auxquelles les propriétaires lésés sont en droit de recevoir une indemnisation suite à la suppression de leur droit de construire (équivalant à une "expropriation matérielle»). Cette judiciarisation du processus de mise en œuvre de la politique de l'aménagement du territoire est le signe d'une incohérence fondamentale entre le SDP (principe de la garantie 
de la propriété) et la PP d'aménagement du territoire, incohérence que les juges - et non le législateur - ont été chargés de résoudre au cas pas cas. Soulignons l'ampleur de leur responsabilité face à la politique naissante de l'aménagement du territoire : une jurisprudence trop favorable aux propriétaires aurait sonné le glas de la politique d'aménagement du territoire. Dans la pratique, c'est la voie opposée qui a été retenue puisque les tribunaux ont choisi de réduire le nombre de situations dans lesquelles une compensation financière des propriétaires par la commune s'avère nécessaire, ceci au travers du durcissement des conditions d'octroi des indemnisations aux propriétaires fonciers négativement affectés par le zonage, choix qui a permis de réduire les risques financiers pesant sur les communes dans le cadre de la mise en œuvre de leurs plans d'affectation. Les critères utilisés par les juges pour élaborer, puis durcir, la jurisprudence proviennent des PP et notamment de la LAT, la jurisprudence fonctionnant ainsi comme une sorte de mécanisme de traduction ou de recombinaison du droit de l'aménagement du territoire (LAT) dans le droit foncier (CC).

L'émergence graduelle, à partir du milieu des années 1980, d'une politique de protection qualitative des sols et des biotopes caractérise la cinquième étape (1983-2007). Plusieurs domaines de PP voient simultanément se développer des dispositions allant dans ce sens : la protection de l'environnement, la politique agricole, la protection de la nature et du paysage ainsi que la protection des eaux. La mise en œuvre de la Loi fédérale sur la protection de l'environnement (LPE, 1983) constitue sans conteste l'événement majeur de cette période. L'objectif principal de la LPE est la lutte contre les atteintes chimiques. Suite à sa révision en 1995, la protection qualitative intègre également la lutte contre les atteintes biologiques et physiques. Pour ce faire, des valeurs limites d'immissions et d'émissions de polluants (avant tout les métaux lourds) dans l'air, des limitations de substances dangereuses pour les sols et l'environnement (engrais, produits pour les plantes, boues d'épuration ou OGM), ou encore des prescriptions relatives à la contamination des sols et de la pollution des eaux par l'entreposage de déchets sont édictées. Les profonds changements que connait la politique agricole à partir du début des années 1990 (abandon du référentiel productiviste et écologisation du système de subventionnement) contribuent également à renforcer la protection qualitative des sols en imposant en 1992-93 de nouvelles limitations aux droits d'usage des agriculteurs dans la zone agricole (épandages, méthodes culturales, etc.).

Ainsi, avec la mise en œuvre de ces diverses politiques publiques, la quasitotalité des biens et services de la ressource sol sont maintenant régulés, directement ou indirectement.

\subsection{L'évolution des RIR du sol}

La combinaison des deux périodisations du SDP et des PP permet de distinguer six configurations successives de régimes institutionnels pour la régulation des usages du sol entre la fin du 19e siècle et aujourd'hui (tableau 3). 
Tableau 3 - Périodisation des RIR du sol en Suisse entre 1870 et 2000

\begin{tabular}{|c|c|c|c|}
\hline Étapes & $\begin{array}{l}\text { Systèrte de droits de propristé } \\
\text { (SDP) }\end{array}$ & $\begin{array}{l}\text { Poiltiques publiques d'expbitation et de protection } \\
\text { (PP) }\end{array}$ & Régimes irstitutionnels du sol (RIR) \\
\hline $\begin{array}{ll}1 . \\
1870 \\
1912\end{array}$ & 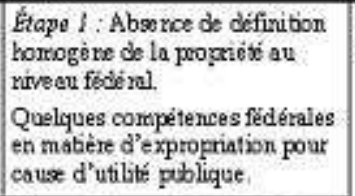 & $\begin{array}{l}\text { Etape 1: Abse roe de toute politique problique } \\
\text { régulant les usages de la ressource sol }\end{array}$ & 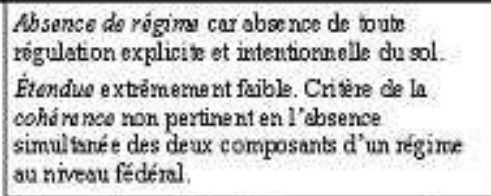 \\
\hline $\begin{array}{l}\text { II. } \\
1912 . \\
1940\end{array}$ & $\begin{array}{l}\text { Etape 2: Introduction du Code } \\
\text { civil, de finition de la propriéxé } \\
\text { au nivesu fedéral, renforce ment } \\
\text { des principes releteff a } \\
\text { l'expropriation. }\end{array}$ & & 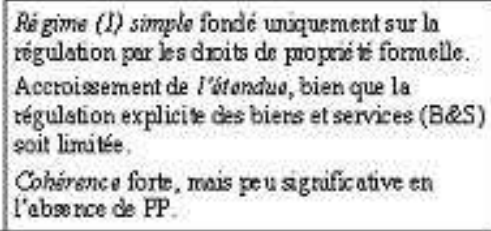 \\
\hline $\begin{array}{l}\text { III. } \\
1940- \\
1955\end{array}$ & $\begin{array}{l}\text { Etapo } 3 \text { : Limitetion des droits } \\
\text { de disposition sur les teres } \\
\text { agricoles }\end{array}$ & 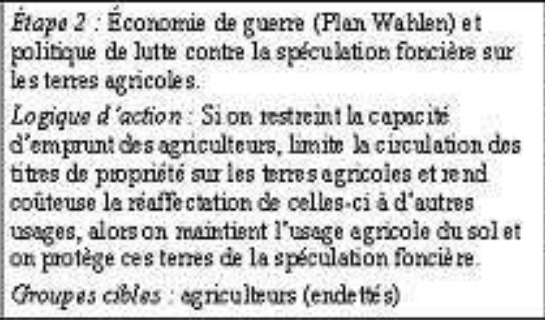 & 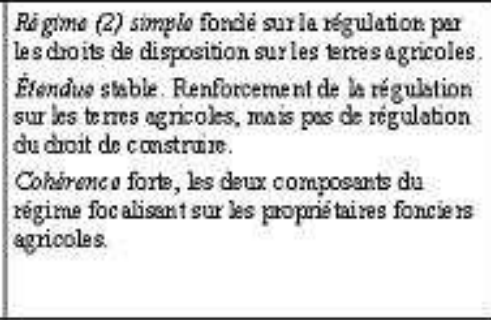 \\
\hline $\begin{array}{l}\text { iv. } \\
1955 . \\
1969\end{array}$ & $\begin{array}{l}\text { Etape } 4 \text { : Premiéres } \\
\text { interventions aménogistes } \\
\text { indiroctos limitant le doit de } \\
\text { construire. }\end{array}$ & 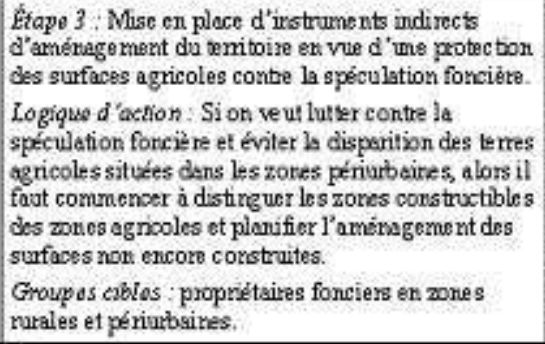 &  \\
\hline \begin{tabular}{|l|}
7. \\
1969. \\
1983
\end{tabular} & 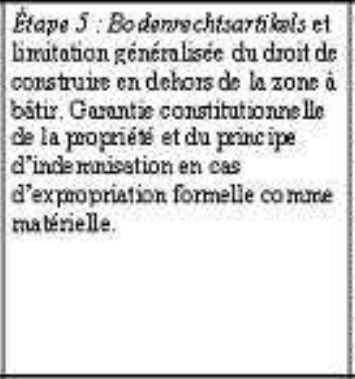 & 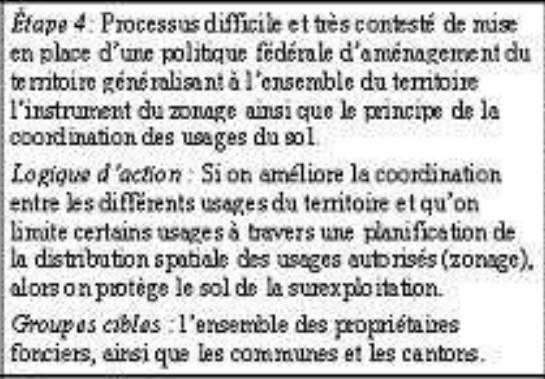 & 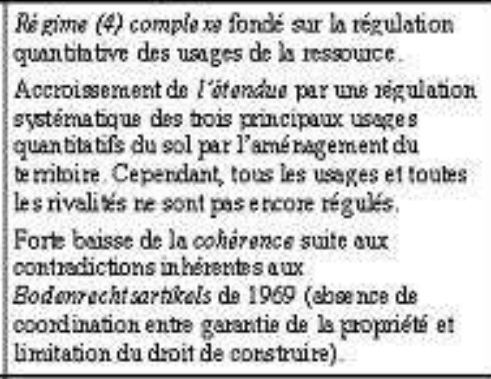 \\
\hline \begin{tabular}{|l|} 
VI. \\
1983. \\
2007
\end{tabular} &  & 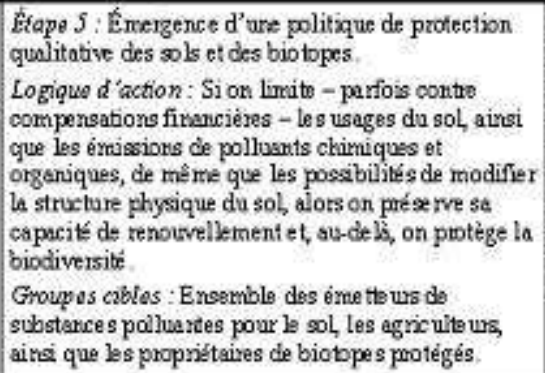 & 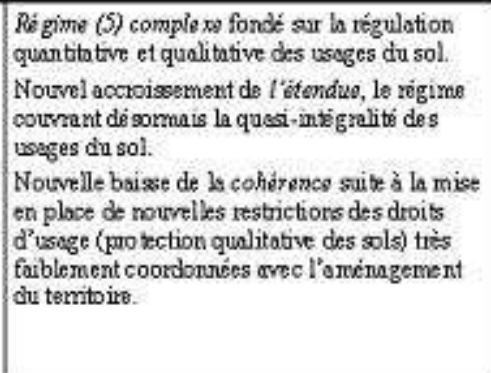 \\
\hline
\end{tabular}


Cette périodisation permet de repérer deux changements majeurs dans la trajectoire historique des régimes du sol. Premièrement, l'introduction du Code civil en 1912 marque le passage d'une situation d'absence de régime à celle d'un régime simple. Secondement, les Bodenrechtsartikels de 1969 institutionnalisent le compromis historique entre la garantie de la propriété et le principe de l'aménagement du territoire, soit le passage d'un RIR simple à un RIR complexe.

Au-delà de l'analyse historique de la trajectoire du RIR du sol en Suisse, plusieurs études de cas détaillées, à un niveau régional ou local, permettent également de démontrer les effets d'un changement de RIR sur la durabilité de la ressource. Sans pouvoir reprendre ici ces éléments évaluatifs en détail, soulignons que l'hypothèse selon laquelle il existe une relation de causalité étroite entre le type de RIR (simple, complexe, intégré) et le niveau de durabilité de la gestion des usages de la ressource régulée a été testée à de multiples reprises, sur différentes ressources (sol, eau, forêt, air, paysage, faune), dans divers contextes nationaux (suisse, français, italien, espagnol, belge et néerlandais) et pour divers périmètres géographiques (national, régional et local). Elle semble largement confirmée par ces premiers tests empiriques (cf. par exemple Reynard 2000 ; Nahrath 2000 ; Varone et al. 2002 ; Knoepfel et al. 2001 et 2003 ; Kissling-Näf et Kuks 2004 ; Bressers et Kuks 2004 ; Rodewald et Knoepfel 2005 ; Gerber 2006).

\section{RIR, TR et développement durable: lectures croisées}

Le cadre théorique des RIR a été développé pour analyser les variables permettant d'expliquer la (non) durabilité des modalités de régulation des usages des ressources, en combinant les approches de l'économie institutionnelle des ressources et de l'analyse des politiques publiques. Quand bien même cette approche n'a pas directement et consciemment puisé d'éléments conceptuels dans la TR, dont les ambitions et le champ d'application sont bien plus larges au demeurant, force est cependant de constater que les deux cadres théoriques ne sont pas incompatibles mais, au contraire, affichent plusieurs points de convergence et de complémentarité. Nous en tentons ici la démonstration en discutant, à partir de notre analyse historique du RIR du sol en Suisse, les propositions que R. Boyer a formulées pour renouveler la TR et qui ont été rappelées en introduction de cet article (cf. note 1). Celles-ci concernent, successivement, (1) la clarification du concept d'institution, (2) la variété des formes de "configurations institutionnelles» (RIR) et des mécanismes qui sous-tendent leurs éventuelles transformations, (3) la viabilité de ces configurations qui découle de leur grande capacité d'adaptation et/ou (4) de l'absence de couplage entre leurs éléments 
constitutifs (SDP et PP) et, finalement, (5) les impacts des changements de ces configurations sur la gestion durable des ressources naturelles.

(1) Le concept d'«institution » est souvent pris dans un sens très extensif dans la TR. Fort de ce constat, R. Boyer (2003:198-199) exhorte les chercheurs régulationnistes à clarifier la nature des règles institutionnelles. De fait, le cadre des RIR procède à cette clarification en identifiant deux types distincts d'institutions, à savoir les droits de propriété (SDP) et les politiques publiques (PP). Plus encore, il permet une analyse systématique des modes d'articulation de différents supports formels de ces deux types de règles institutionnelles : les principes constitutionnels, le droit public (législations fédérales, cantonales et locales), le droit privé (CC, Code des obligations), le droit administratif et la jurisprudence des tribunaux. Il analyse également le rôle respectif joué par ces règles institutionnelles plurielles au sein d'une configuration institutionnelle historique spécifique (RIR). Ce faisant, il permet de distinguer les différents droits d'usage et (groupes d') usagers d'une ressource sous l'angle de la robustesse de ces droits, selon que ceux-ci sont fondés dans le SDP, dans les $\mathrm{PP}$ et/ou dans la jurisprudence y relative. Une telle distinction est, à notre sens, fort utile - aussi bien dans une perspective empirico-analytique que normative - dans la mesure où elle permet d'expliquer et, en même temps, d'anticiper leurs capacités respectives de résistance sociopolitique.

Notons aussi que le cadre des RIR, en focalisant en premier lieu son attention sur les règles institutionnelles formelles, permet de distinguer clairement ces dernières des régulations informelles (par exemple des normes sociales qui influencent fortement le comportement des usagers d'un ressource naturelle), ne serait-ce qu'en contextualisant le cadre formel au sein duquel ces régulations informelles émergent, perdurent, se transforment ou disparaissent. Plusieurs travaux empiriques démontrent par exemple comment, en cas de rivalités d'usage entre différents acteurs, ces derniers recourent soit au SDP et/ou aux $\mathrm{PP}$ soit à des arrangements purement informels pour résoudre leurs différends (voir Aubin 2007 pour une analyse des conflits entre usagers de la ressource eau). L'approche des RIR ne nie donc nullement l'importance des régulations informelles mais envisage leur analyse en creux plutôt qu'en relief.

(2) Relu dans la perspective de la TR, le concept de RIR n'est, en soi, rien d'autre qu'un cadre d'analyse permettant de rendre compte de l'évolution historique des «configurations institutionnelles » successives régulant les multiples usages des ressources naturelles. Il permet de développer des démarches comparatives aussi bien diachroniques (comparaison des changements au sein d'un ou de plusieurs RIR à différentes périodes du temps) que synchroniques (comparaison des changements de RIR dans différents pays et à différentes échelles institutionnelles) concernant les effets des (changements de) configurations institutionnelles (cf. RIR simples, complexes ou intégrés) sur la (non) durabilité de la gestion des ressources. En ce sens, il s'inscrit parfaitement dans la perspective de l'approche historique comparative dont la pertinence est revendiquée par R. Boyer (2003:198) dans la mesure où il permet de «révéler la variété des formes d'évolution» des institutions (cf. processus 
de conversion, sédimentation, recombinaison). En effet, l'analyse du RIR du sol en Suisse a bien mis en lumière cette diversité des dynamiques de changements institutionnels (cf. tableau 3), que nous réinterprétons ici à l'aide des catégories proposées par R. Boyer (2003:187 ss).

L'entrée en vigueur du CC en 1912 constitue le moment fondateur du SDP actuel (droit foncier) du RIR du sol et peut être interprété comme la création d'une nouvelle institution au travers d'un processus de sédimentation (par changement d'échelle), le nouveau CC fédéral venant se superposer aux codes civils cantonaux existants. Ce faisant, il opère une sorte de synthèse sélective des principaux éléments des différentes traditions (française et germanique) présentes dans les CC cantonaux.

Le développement des différentes PP à incidences spatiales à partir des années 1950-1960, soit successivement les politiques agricole, de protection de l'eau, de protection de la nature et du paysage, puis finalement d'aménagement du territoire, peut être clairement interprété comme un vaste processus de sédimentation de dispositions de droit public venant redéfinir - le plus souvent sous la forme de limitations - le contenu des droits d'usage des propriétaires fonciers, ainsi que, à partir des années 1980, de l'ensemble des usagers du sol. Par sédimentation des dispositions de PP, il convient de comprendre aussi bien la superposition au cours du temps des différentes logiques d'action successives au sein d'une seule et même politique sectorielle (sédimentation interne), que la rencontre - plus ou moins coordonnée et cohérente - au cours du temps entre les différents modèles d'intervention des diverses PP à incidences spatiales (sédimentation externe). Ce processus généralisé de sédimentation externe peut, dans certains cas, mener à des phénomènes de conversion d'une politique sectorielle : citons, par exemple, l'écologisation de la politique agricole à partir du début des années 1990 sous la pression des politiques environnementales dont la protection des eaux et des sols.

Mais l'impact le plus important de la montée en puissance des dispositions régulatrices des PP consiste certainement dans le déclenchement d'un premier processus de recombinaison du RIR du sol sous la forme des articles constitutionnels sur le droit foncier (Bodenrechtsartikels) de 1969. Ces dispositions du SDP viennent consacrer le principe d'équivalence entre la garantie de la propriété privée et la défense de l'intérêt public. Cependant la situation d'incohérence résultant de cette première recombinaison entre politique d'aménagement du territoire et droit foncier a nécessité la production d'une intense jurisprudence en matière d'indemnisation pour cause d'expropriation matérielle (soit la compensation financière, par l'Etat, des propriétaires fonciers dans le cas où l'usage de leur bien-fonds est limité pour des raisons d'utilité publique, par exemple la réalisation d'une infrastructure publique de transport ou la protection de l'environnement). Les choix effectués par le Tribunal fédéral (qui est l'instance judiciaire suprême en Suisse) durant les années 1970 consistant à durcir les conditions de reconnaissance de la situation d'expropriation matérielle, ceci de manière à rendre possible la mise en œuvre des instruments de planification de la politique d'aménagement du 
territoire, a mené, d'une part, à un second processus de recombinaison de la configuration institutionnelle du RI du sol consacrant, cette fois, la primauté de l'objectif de la planification (politiques publiques) sur celui de la protection absolue des intérêts des propriétaires fonciers (SDP). D'autre part, on observe également une véritable conversion de l'institution de l'expropriation matérielle dans la mesure où l'objectif initial consistant dans la garantie de la propriété foncière a été remplacé par la création des conditions juridiques et économiques nécessaires à la mise en œuvre de la politique d'aménagement du territoire.

(3) L'analyse historique du RIR du sol en Suisse démontre également la pertinence de la proposition de R. Boyer (2003:197) selon laquelle « la viabilité d'une institution ne tient pas à la permanence de chacun de ses traits, mais à la capacité générative de la configuration correspondante et aux potentialités d'adaptation à des contextes variables dans le temps et aussi dans l'espace ». Le cas analysé ici démontre à la fois la robustesse de l'institution de la propriété privative, telle qu'elle est définie dans l'article 641 du CC, et la capacité d'adaptation de celle-ci à un contexte évolutif. Ainsi, les défenseurs du droit foncier sont parvenus à conserver relativement inchangés, durant près d'un siècle, les fondements philosophiques et juridiques de cette institution, par-delà les bouleversements des contextes sociaux, politiques, économiques et environnementaux qui jalonnent l'histoire mouvementée du $20^{\mathrm{e}}$ siècle. De fait, la propriété foncière est successivement passée d'une situation où elle jouissait du quasi monopole de la régulation des usages du sol à une situation où les pressions diverses sur les terres agricoles (économie de guerre, spéculation, urbanisation) ont nécessité de la reconfigurer au travers de régulations spécifiques pour les terres agricoles et les espaces ruraux (droit foncier rural, zones agricoles et protégées), puis pour les zones constructibles (police des construction, zone à bâtir). En un mot, l'institution du droit foncier a réussi à s'adapter aux fortes pressions résultant de la montée en puissance des PP à partir des années 1950 et 1960, sans pour autant connaittre de transformations significatives de ses principes fondamentaux.

Un autre exemple emblématique de la capacité d'adaptation de l'institution de la propriété concerne l'introduction de la propriété par étage (PPE) dans le CC en 1963. Il s'agit là d'une reformulation partielle et modernisée de l'ancienne institution de la propriété commune ("common property») qui existait dans les anciens CC cantonaux, mais qui avait été supprimée au moment de l'entrée en vigueur du CC fédéral en 1912. Cette modification adapte l'institution de la propriété à une nouvelle contrainte contextuelle consistant dans la nécessité de faciliter l'accès d'une plus grande part de la population à la propriété immobilière en temps de crise du logement et de renchérissement important du prix du sol et des loyers. Ce second exemple met en lumière la capacité de résilience d'une forme ancienne de conception de la propriété, sa réapparition correspondant à une forme de transformation institutionnelle - qui n'est cependant pas envisagée explicitement par R. Boyer - à savoir la résurgence 
sédimentaire (ajout de l'ancien à du neuf), qui correspond à une sorte d'inversion du processus de sédimentation classique (ajout du neuf à l'ancien).

En conclusion, l'évolution du RIR du sol en Suisse confirme l'hypothèse de R. Boyer dans la mesure où la viabilité de l'institution de la propriété privative (SDP) est liée à la capacité générative de la configuration institutionnelle (RIR) qu'elle forme avec la politique publique d'aménagement du territoire (PP). La mise en place du compromis de 1969 (Bodenrechtsartikels) et le développement de la jurisprudence en matière d'expropriation matérielle constituent deux mécanismes générant des solutions institutionnelles permettant d'adapter l'institution de la propriété foncière aux nouvelles exigences de l'aménagement $\mathrm{du}$ territoire sans renoncer aux principes fondamentaux de celle-ci. Si les Bodenrechtsartikels constituent, dans un premier temps, une manière de soumettre le principe de l'aménagement du territoire à l'exigence de la garantie de la propriété, la jurisprudence fédérale en matière d'indemnisation pour expropriation matérielle consacre, quant à elle, un mouvement inverse d'adaptation, par les tribunaux, du droit foncier aux principes, objectifs et instruments de l'aménagement du territoire. Force est de constater que malgré son caractère casuel et provisoire, cette configuration institutionnelle a jusqu'ici relativement bien réussi à «stabiliser le jeu des acteurs, fût-il conflictuel et contradictoire » (Boyer 2003 :197), et ce malgré la complexité du RIR en place.

(4) En effet, tout se passe comme si l'incohérence du "RIR complexe » actuel représente, non pas un facteur d'affaiblissement de la configuration institutionnelle de régulation du sol mais, au contraire, un facteur de renforcement (en tout cas temporaire) de celle-ci. Pareil constat confirme la pertinence d'un argument supplémentaire de R. Boyer (2003:198) selon lequel « la viabilité d'une configuration ne [résulte] pas nécessairement de l'intensité du couplage entre les éléments constituants. Il se pourrait en effet qu'une intégration imparfaite suscite un flou et une incertitude quant à l'application des règles du jeu dont peuvent s'emparer les acteurs en vue d'innover et éventuellement de forger de nouvelles configurations ». Ainsi, la retenue du législateur suisse qui n'a pas légiféré sur la question de la définition de l'expropriation matérielle ainsi que son choix de déléguer cette tâche aux tribunaux ont été interprétés par certains commentateurs de la jurisprudence (Moor 1982, 2002) comme l'expression d'un refus délibéré d'une définition juridique par trop « théorique » et rigide, au profit d'une définition empirique et casuistique, c'est-à-dire adaptable aux évolutions contextuelles. C'est précisément ce que semblent attester les inflexions récentes de la jurisprudence, en direction d'un renforcement de la protection des intérêts des propriétaires fonciers cette fois ci, suite au changement de situation juridique créé par l'existence, depuis les années 1980, des premiers plans d'affectations conformes au droit fédéral au sein desquels la définition de la zone à bâtir (souvent surdimensionnée) peut désormais être invoquée par les propriétaires cherchant à garantir leur droit à bâtir contre les autorités publiques cherchant elles à réduire les portions surdimensionnées de leur zone à bâtir (cf. Nahrath 2005 :316-317). 
(5) Les mécanismes qui sous-tendent les changements institutionnels, tels qu'ils ont été proposés par R. Boyer (cf. processus de sédimentation, conversion et recombinaison), permettent d'affiner l'analyse des dynamiques et évolutions qui caractérisent les trajectoires historiques des RIR. L'approche en termes de RIR se concentre, quant à elle, sur le contenu des configurations institutionnelles. Elle vise clairement à élaborer une typologie des régimes institutionnels de ressource (cf. RIR inexistant, simple, complexe et intégré), en fonction de la cohérence et de l'étendue des modalités de régulation des usages, et, par-là, à identifier l'ampleur des changements de RIR observés dans le temps. De plus, le cadre des RIR propose une hypothèse centrale sur l'impact des régimes institutionnels sur la durabilité de la gestion des ressources naturelles: plus un RIR est intégré, plus il favorise une gestion durable de la ressource; à l'inverse, plus il est simple (voire inexistant), plus le risque d'une surexploitation généralisée de la ressource augmente. Force est donc de souligner la complémentarité évidente entre la TR et le cadre des RIR. Le couplage de ces deux approches permet en effet d'établir un lien théorique entre les changements institutionnels, le contenu des RIR qui en découle et leurs effets sur la durabilité de la gestion des ressources naturelles.

Sur la base de notre analyse empirique du sol en Suisse, nous formulons ainsi l'hypothèse de recherche suivante, qui croise explicitement les propositions théoriques de la TR et du cadre des RIR : la gestion durable d'une ressource naturelle n'est assurée que si le RIR est intégré, et un RIR complexe ne tend vers l'intégration que si un processus de recombinaison implique une rehiérarchisation de ses composants (SDP et PP), lesquels connaissent conjointement une conversion qui garantit leur mise en cohérence. Concrètement, nous arguons que seul un nouvel ordonnancement entre l'institution de la propriété privée et les politiques à incidences environnementales, dont l'aménagement du territoire en premier lieu, ainsi qu'un renforcement de l'expropriation matérielle - compris ici comme une conversion de l'institution de la propriété privée - permettraient une mise en cohérence des composants du RIR du sol en Suisse, donc son évolution vers un régime intégré et, par-là, vers une gestion durable du sol.

Par défaut, cette hypothèse stipule aussi que les changements institutionnels par sédimentation induisent des processus d'adaptation trop limités pour garantir la durabilité à long terme de la gestion d'une ressource naturelle, surtout si celle-ci connaît une croissance des rivalités d'usage. Il convient toutefois de rappeler ici que des RIR complexes ne garantissant nullement une gestion durable peuvent parfaitement s'avérer robustes, en raison même de leur relative incohérence (cf. point 4 ci-dessus). La stabilité du RIR complexe du sol en Suisse en atteste empiriquement.

\section{Conclusion}

A notre avis, les cinq points mentionnés ci-dessus mériteraient un débat entre les tenants du RIR et ceux de la TR plus rigoureux et étendu que celui 
sommairement amorcé ici. Tout semble en effet indiquer que le dialogue pourrait s'avérer fructueux. Preuve en est l'intérêt marqué - pour le cadre des RIR - des diverses propositions émises par R. Boyer sur la hiérarchie et la complémentarité institutionnelle, propositions qui s'avèrent globalement confirmées par notre propre analyse historique du RIR du sol en Suisse. De même, gageons que la prise en compte du cadre analytique des RIR par les chercheurs régulationnistes favoriserait une opérationnalisation de plusieurs concepts macro et hypothèses générales de la TR, ainsi que leur application empirique à la gestion des ressources naturelles et au DD.

Afin de pouvoir élaborer, ensemble, une véritable grammaire des configurations institutionnelles, de leurs composants, mécanismes de changements et effets sur le DD, deux autres dimensions devront être abordées préalablement. La première concerne le périmètre de régulation pertinent pour le DD et, par conséquent, les échelles de l'analyse. Alors que la logique institutionnelle plaide évidemment pour retenir les territoires des entités politiques au sein desquels les configurations institutionnelles émergent, sont mises en œuvre et déploient leur effets (par exemple le champ d'application national pour le Code civil ou communal pour des plans d'aménagement territorial), la logique des ressources naturelles invite quant à elle à retenir des «espaces fonctionnels» (Nahrath et Varone 2007) qui font immédiatement sens en termes de durabilité (par exemple le bassin versant transnational d'un fleuve ou une forêt locale). La seconde prémisse à préciser concerne la place et le rôle des acteurs, à savoir les usagers des ressources naturelles, pour lesquels les configurations institutionnelles représentent aussi bien des opportunités que des contraintes. La prise en compte de leurs intérêts et répertoires d'action, ainsi que des idées, matrices cognitives ou «visions du monde» (Boyer 2003 :173) qu'ils défendent, est indispensable à la compréhension non seulement des changements institutionnels mais aussi des effets de ceux-ci sur la durabilité de la gestion des ressources naturelles.

\section{Bibliographie}

Aubin, David (2007), L'eau en partage: Activation des règles dans les rivalités d'usages en Belgique et en Suisse, PIE-Peter Lang, Bruxelles.

Bisang, K. et Schenkel, W. (2003), "Effekte des regionalen Regimewandels der Ressource Wald", in Knoepfel, P., Kissling-Näf, I. et Varone, F. (Editeurs), Institutionelle Ressourcenregime in Aktion - Régimes institutionnels de ressources naturelles en action. Helbing \& Lichtenhahn, Basel, pp. 141-204.

Boyer, R. (2003), «Les analyses historiques comparatives du changement institutionnel : quels enseignements pour la théorie de la régulation? ", L'Année de la régulation, "Économie, Institutions, Pouvoirs", 7: 167-203. 
Bressers, H. et Kuks, S. (Editeurs) (2004), Integrated Governance and Water Basin Management. Conditions for Regime Change and Sustainability, Kluwer Academic Publishers, Dordrecht.

Bromley, D. (1991), Environment and Economy. Property Rights and Public Policy. Blackwell, Oxford.

Gerber, J.-D. (2006), Structures de gestion des rivalités d'usage du paysage. Une analyse comparée de trois cas alpins, Rüegger Verlag, Zürich/Chur.

Jessop, B. (1997), "The Regulation Approach", Journal of Political Philosophy, 5: 287-326.

Kirat, Th. (2005), «Les conflits liés au voisinage. L'effet des relations juridiques sur la construction institutionnelle de l'espace», in Torre, A. M. Filippi (coord.), Proximité et changements socio-économiques dans les mondes ruraux, INRA, Paris, pp.243-256.

Kirat, Th. et R. Melot (2006), «Du réalisme dans l'analyse économique des conflits d'usage. Les enseignements de l'étude du contentieux dans trois départements français (Isère, Loire-Atlantique, Seine-Maritime) », Développement durable et territoire, Dossier 7: proximité et environnement, http://developpementdurable.revues.org/document2574.html (consulté le 9 octobre 2007).

Kissling-Näf, I. et Kuks, S. (Editeurs) (2004), The Evolution of National Water Regimes in Europe. Transitions in water rights and water policies. Kluwer Academic Publishers, Dordrecht.

Kissling-Näf, I. et Varone, F. (Editeurs) (2000), Institutionen für eine nachbaltige Ressourcennutzung: Innovative Steuerungsansätze am Beispiel der Ressourcen Luft und Boden, Rüegger, Zurich.

Knoepfel, P., Kissling-Näf, I. et Varone, F. (Editeurs) (2001), Institutionelle Regime für natürliche Ressourcen: Boden, Wasser und Wald im Vergleich - Régimes institutionnels de ressources naturelles: analyse comparée du sol, de l'eau et de la forêt, Helbing \& Lichtenhahn, Basel.

Knoepfel, P., Kissling-Näf, I. et Varone, F. (Editeurs) (2003), Institutionelle Ressourcenregime in Aktion / Régimes institutionnels de ressources naturelles en action, Helbing \& Lichtenhahn, Basel.

Knoepfel, P., Nahrath S. et Varone, F. (2007), "Institutional Regimes for Natural Resources: An Innovative Theoretical Framework for Sustainability", in Knoepfel, P., Environmental Policy Analyses. Learning from the Past for the Future 25 Years of Research, Berlin, Springer, pp.455-506.

McNeill, J.R. (2001), Something New Under the Sun: An Environmental History of the Twentieth-Century World. Norton, New York. 
Moor, P. (1982), «Aménagement du territoire et expropriation matérielle: l'évolution de la jurisprudence du Tribunal fédéral ", Repertorio du giurisprudenza patria, n 115 , vol.5, pp.270-286.

Moor, P. (2002), "L'expropriation matérielle », in Droit administratif, vol. II, Berne, Stämpfli, pp.741-755.

Nahrath, S. (2000), "“Governing Wildlife Resources?” L'organisation de la chasse en Suisse comme exemple de régimes institutionnels de gestion d'une ressource naturelle », Revue Suisse de Science Politique, 6 : 123-158.

Nahrath, S. (2003), "Les effets de la mise en place du régime de l'aménagement du territoire sur les rives du lac de Bienne, la colline du « Hueb » et dans l'agglomération lausannoise entre 1960 et 1990 », in Knoepfel, P., Kissling-Naef, I., Varone, F. (Editeurs), Institutionelle Ressourcenregime in Aktion / Régimes institutionnels de ressources naturelles en action, Helbing \& Lichtenhahn, Basel, Genf, München, pp. 9-140.

Nahrath, S., 2005. «Le rôle de la propriété foncière dans la genèse et la mise en œuvre de la politique d'aménagement du territoire », in: Da Cunha, A., Knoepfel, P., Leresche, J.-P., Nahrath, S. (Editeurs), Enjeux du développement urbain durable. Transformations urbaines, gestion des ressources et gouvernance. Presses Polytechniques et Universitaires Romandes, Lausanne, pp. 299-328.

Naharath, S. et Varone, F. (2007), «Les espaces fonctionnels comme changement d'échelles de l'action publique », in Faure, A., Leresche J.-Ph., Muller P., Nahrath S. (Editeurs) Action publique et changements d'échelles: les nouvelles focales du politique, L'Harmattan (coll. Logique politique), Paris, pp. 235249.

Organisation de Coopération et de Développement Economiques (OCDE), (2007), Examens environnementaux de l'OCDE - Suisse (2007), OCDE, Paris.

Ostrom, E. (1990) Governing the Commons: the Evolution of Institutions for Collective Action. Cambridge University Press, Cambridge.

Reynard, E., 2000, "Cadre institutionnel et gestion des ressources en eau dans les Alpes : deux études de cas dans des stations touristiques valaisannes », Revue Suisse de Science Politique, 6 : 53-85.

Rodewald, R. et Knoepfel, P. (Editeurs) (2005), Institutionelle Regime für nachbaltige Landschaftsentwicklung - Régimes institutionnels pour le développement durable du paysage, Rüegger, Zurich.

Steiger, O. (2006), "Property Economics versus New Institutional Economics: Alternative Foundations of How to Trigger Economic Development", Journal of Economic Issues, 40: 183-208.

Thelen, K. (2003), «Comment les institutions évoluent:. Perspectives de l'analyse comparative historique ». L'Année de la régulation, "Économie, Institutions, Pouvoirs", 7: 13-43. 
Revue de la régulation, Capitalisme, Institutions, Pouvoirs, $n^{\circ} 2,2008$ http ://regulation.revues.org

Varone, F., Reynard, E., Kissling-Näf, I. and Mauch, C., 2002. "Institutional Resource Regimes. The case of water in Switzerland", Integrated Assessment, 3: 78-94.

WCED (1987), Our Common Future, Oxford University Press, Oxford. 